\title{
Les nouvelles dynamiques intra-métropolitaines de l'emploi favorisent-elles des migrations pendulaires plus « durables »? le cas de la région métropolitaine de Montréal, 1998-2003
}

\section{Do new intra-metropolitan employment dynamics generate more "sustainable » commuting? The case of Montreal metropolitan area, 1998-2003}

\author{
Rémy Barbonne, Richard G. Shearmur, William J. Coffey \\ INRS-Urbanisation, Culture et Société, \\ 385, rue Sherbrooke Est, Montréal, Québec, Canada H2X $1 E 3$ \\ Département de géographie, Université de Montréal, C.P. 6128, Succ. CV \\ Montréal, Québec, Canada H3C 3J7
}

\section{Résumé}

Utilisant un découpage extrêmement fin et détaillé des zones d'emplois de la région métropolitaine de Montréal, cette étude vise à mieux cerner l'évolution (1) des distances de navette résidence-travail et (2) des modes de transport associés aux nouvelles dynamiques intra-métropolitaines de l'emploi (Polynucléarisation vs. dispersion). Pour ce faire nous exploitons les données de déplacements pour le travail issues des enquêtes origine-destination de 1998 et 2003 de l'Agence métropolitaine de Transport (AMT) de Montréal. Nos résultats indiquent clairement que, si la polynucléarisation de l'emploi va de pair avec l'adoption de comportements de mobilité moins « durables », l'utilisation d'une classification plus raffinée des zones d'emploi, distinguant notamment entre les noyaux et les franges des principaux pôles d'emploi révèle que cette tendance est largement contrebalancée par les nouvelles formes d'étalement de l'emploi autour de ces pôles auxquelles sont associés des comportements de mobilité plus « durables ».

(C) 2008 Lavoisier, Paris. Tous droits réservés. 


\begin{abstract}
Summary
Using a very detailed and disaggregated classification of job location, this study aims at better describing the evolution of commuting distances and mode choice associated with new forms of employment distribution in the Montreal metropolitan region (polynucleation vs. scatteration). For this purpose, we use the 1998 and 2003 OD survey of the Montreal Metropolitan Transport Agency. Our results clearly underline the fact that if polynucleation is associated with less "sustainable" commuting, the use of a more detailed classification of employment zones, distinguishing between core and frame of the most important job centers, sheds light on the fact that this trend is, to a large extent, offset by new forms of employment diffusion around these job centers, which are associated with more "sustainable" commuting.

(C) 2008 Lavoisier, Paris. Tous droits réservés.
\end{abstract}

Mot clés : emploi, intra-métroploitain, polynucléarisation, dispersion, navettes, distances, modes de transport.

Keywords : employment, intra-metropolitan, polynucleation, scatteration, journey-to-work, commuting distances, transport mode.

\title{
Introduction
}

Dans un contexte de volonté de réduction des gaz à effet de serre, la problématique des déplacements des individus est devenue de plus en plus cruciale. Parmi ces déplacements, les navettes résidence-travail (migrations pendulaires) sont probablement les plus déterminantes, notamment parce qu'elles structurent la vie quotidienne des individus et qu'autour d'elles s'organisent bon nombre de leurs activités et de leurs déplacements pour d'autres motifs (Kwan, 1999; Dielman et al. 2002). Malgré l'importance que revêt une meilleure connaissance de ces déplacements, on peut déplorer que cette question ait été essentiellement abordée par des géographes sociaux, mettant plutôt l'accent sur les caractéristiques des individus ou de leur quartier de résidence, comme en témoigne l'abondante littérature visant à tester empiriquement les mesures préconisées par les tenants du nouvel urbanisme (Ewing et Cervero, 2001). Plus rares sont les géographes économiques qui se sont intéressés à cette question en considérant explicitement et distinguant les différents types de lieux d'emploi des travailleurs (Cervero, 1997a, 1998; Sultana, 2000; Aguilera, 2005; Shearmur 2006). Néanmoins, le découpage généralement utilisé par ces auteurs ainsi que les critères d'identification des pôles d'emploi retenus dans leurs analyses manquent souvent de finesse et d'exhaustivité pour prendre en compte les nouvelles dynamiques spatiales de l'emploi, particulièrement celles relevant des formes polynucléaires ou de dispersion généralisée (scatteration) associées au processus de desserrement de l'emploi ayant affecté les métropoles au cours des dernières décennies. Désireux de pallier cette lacune, nous recourons dans cette étude à un découpage particulièrement fin et détaillé des zones d'emploi utilisé dans une précédente recherche portant sur l'évolution entre 1996 et 2001 de la répartition intra-métropolitaine de l'emploi au sein de la région métropolitaine de recensement (RMR) de Montréal (Shearmur et al, 2007) afin d'analyser le plus finement possible l'évolution entre 1998 et 2003 (1) des distances parcourues ainsi que (2) des modes de transports utilisés par les travailleurs de cette région. Nos 
résultats témoignent de toute la pertinence de recourir à pareil type de découpage, distinguant notamment entre les noyaux et les franges des principaux pôles d'emploi, les comportements de mobilité qui leur sont associés se révélant de façon étonnante sensiblement différents. Mais avant d'élaborer davantage sur ce point, revenons dans un premier temps sur les principales caractéristiques de ces nouvelles dynamiques intra-métropolitaines de l'emploi avant de recenser, dans un second temps, ce que nous apprend la littérature sur les conséquences de ces nouvelles dynamiques spatiales de l'emploi sur l'évolution des déplacements résidence-travail, notamment l'évolution des distances parcourues et des modes de transport utilisés par les travailleurs.

\section{Nouvelles Dynamiques Intra-Métropolitaines De L'Emploi : Polynuclérisation Ou/Et Dispersion Généralisée?}

Le déclin des centres-villes auquel on assiste ne correspond, le plus souvent, qu'à une diminution de son poids relatif dans la hiérarchie des pôles. En effet, dans la majorité des cas la localisation au centre-ville demeure la plus attractive pour les activités hautement spécialisées nécessitant beaucoup de contacts "face à face" (Polèse et Chapain, 2000). Ceci dit, la plupart des chercheurs, constatant le phénomène de déconcentration de l'emploi qui affecte les métropoles occidentales, tant en Amérique du Nord (Gordon et al., 1989; Guiliano et Small, 1991; McDonald et Prather, 1994; Fuji et Hartshorn, 1995; Bogart et Ferry, 1999) qu'en Europe (Aguilera et Mignot, 2002; Aguilera, 2005 ; Naess et Sandberg, 1995 ; Schwanen et al., 2001, 2004) ou encore en Océanie (Pfister et al., 2000 ; Buchanan et al. 2006), s'entendent pour reconnaître que l'on a assisté à un dépassement du modèle monocentrique classique tel que théorisé par Burgess (1925) et modélisé par Alonso (1964).

En revanche, des incertitudes demeurent quant aux nouvelles formes d'organisation spatiale générées par ce desserrement intra-métropolitain de l'emploi. D'un côté, certains auteurs ont souligné dès les années 80 l'apparition d'une nouvelle vague de suburbanisation de l'emploi incluant non plus seulement les services à la population, les activités manufacturières et certaines activités de bureau ne demandant pas de relation face à face et ayant généralement un caractère standardisé et routinier, mais également les services du tertiaire supérieur : "high-order services » et «front-office functions ». On assistait donc à un phénomène de polynucléarisation de l'emploi, c'est-à-dire à une réagglomération de l'emploi décentralisé dans un nombre limité de pôles suburbains diversifiés, allant même jusqu'à héberger des fonctions traditionnellement dévolues au centre-ville (CBD). Cette réagglomération de l'emploi dans des pôles traduisait ainsi la recherche d'économies d'agglomération, telles que celles générées par les centres-villes traditionnels. De ces constats découlent les concepts de «edge cities » (Garreau, 1991), « suburban downtowns » (Hartschorn et Muller, 1989), "suburban employment centers » (Cervero, 1989) ou encore de " magnet areas » (Stanback, 1991). D'un autre coté, certains auteurs ont affirmé dès le milieu des années 1990 (Gordon et Richardson, 1996) que la polynucléarisation serait une étape transitoire vers un mouvement de dispersion généralisée ( «scatteration ») de l'emploi à des densités relativement faibles sur le territoire. Dans la même lignée, Lang (2003) proposait plus récemment le concept de « edgeless cities » pour décrire cette nouvelle forme de diffusion spatiale, d'étalement des bureaux, caractérisée ni par la densité ni par les frontières 
claires que l'on retrouve dans les « edges cities ». Plus radicalement encore, certains auteurs (Dear et Flusty, 2001) à travers leur concept de « keno capitalism » soulignent l'organisation de plus en plus chaotique et empreinte de hasard de l'espace économique urbain, sous l'influence du capitalisme global et du développement des nouvelles technologies de l'information et de la communication, rendant du même coup quasiment vaine toute tentative de mise en lumière de « régularités » spatiales.

Au Canada, plusieurs études (Coffey et Shearmur, 2001, 2002; Shearmur et Coffey, 2002a, 2002b) constatent plutôt une tendance à la polynucléarisation de l'emploi au sein des principales métropoles. En effet, à l'exception du cas de Toronto, on ne pouvait pas vraiment parler d'un phénomène de dispersion généralisée de l'emploi, mais plutôt de renforcement du poids relatif des pôles, voire même du centre-ville, comme dans le cas de Montréal. Cependant, une étude plus récente (Shearmur et al., 2007), recourant à un découpage plus fin (secteurs de dénombrement) et à une méthodologie plus raffinée d'identification des « concentrations » d'emploi, distinguant notamment entre le noyau et les franges des principaux pôles d'emploi, a permis de mettre en lumière au sein de la région métropolitaine de Montréal un phénomène d'étalement, de diffusion de l'emploi dans les pourtours des principaux pôles d'emploi, notamment le long des principaux axes de communication (Terral et Shearmur, 2007). Ces derniers résultats attestent de l'importance de l'échelle d'analyse des dynamiques spatiales de l'emploi et témoignent du fait que la polynucléarisation et certaines formes de dispersion de l'emploi semblent bel et bien pouvoir coexister. Mais voyons maintenant ce que nous apprend la littérature sur les conséquences de ces nouvelles dynamiques spatiales de l'emploi sur l'évolution des déplacements résidence-travail, notamment l'évolution des distances parcourues et des modes de transport utilisés par les travailleurs.

\section{Desserrement de l'emploi et migrations pendulaires : augmentation des distances parcourues et recours accru à l'automobile}

Certains auteurs (Gordon et Richardson, 1991) ont affirmé que la déconcentration intra-métropolitaine de l'emploi avait pour conséquence de rendre les navettes résidencetravail plus courtes, les emplois ayant tendance à se rapprocher de la population qui elle-même s'est largement déconcentrée sur le territoire. Cette thèse, dite de la « colocalisation », peut être rapprochée selon nous aux théories du nouvel urbanisme, et plus particulièrement à son volet qui traite de ses implications en matière de gestion des transports et des comportements de mobilité : le Transit-Oriented-Development (TOD). Les tenants de ce courant de pensée, inspirés par le modèle de la « ville compacte » que représente la traditionnelle ville monocentrique, préconisent en substance de renforcer la densification des pôles suburbains, de favoriser la mixité des usages en leur sein (emplois, commerces, logements), de revoir l'aménagement physique de leur site (concept de «pedestrian pocket ») et de renforcer leur intégration au sein des réseaux de transport en commun afin de favoriser une réduction des distances parcourues ainsi que le recours à des modes de transports alternatifs à l'automobile (transport en commun et transports actifs).

Si plusieurs études empiriques ont effectivement établi que le desserrement de l'emploi pouvait conduire à une stabilisation, voire une réduction dans certains cas, des temps de déplacements dans les métropoles américaines (Dubin, 1991; Guiliano et Small, 1993; 
Wachs et al., 1993; Levinson, 1998; Sultana, 2000), les chercheurs s'accordent, en revanche, à reconnaître que le desserrement de l'emploi favorise plutôt une augmentation des distances parcourues par les travailleurs, et cela, tant en Amérique du Nord (Cervero, 1993; Rosseti et Eversole, 1993 ; Cervero et Wu, 1998) qu'en Europe (Naess et Sandberg, 1995; Aguilera et Mignot, 2002; Schwanen et al., 2004). Or, d'un point de vue environnemental, l'augmentation des distances de navette constitue un enjeu plus important que celle des temps, notamment en raison de ses impacts en matière de pollution urbaine et de coûts relatifs aux infrastructures de transport. Cette tendance s'expliquerait notamment du fait d'une augmentation des déplacements à travers la ville, d'une banlieue à l'autre, pour lesquels les infrastructures routières ne sont pas toujours adaptées. Schwanen et al. (2004) fournissent par ailleurs une bonne synthèse des multiples facteurs pouvant expliquer pourquoi l'hypothèse de colocalisation ne se vérifie généralement pas ; mais disons, qu'en substance, cette controverse pose plus largement la question de l'articulation entre les marchés fonciers et les marchés locaux de l'emploi qui est celle de l'appariement spatial (spatial mismatch) entre les emplois et la population active (Kain, 1968; Preston et McLafferty, 1999; Houston, 2005). La littérature évoquée précédemment nous apprend également plus spécifiquement que les navettes vers les principaux pôles d'emploi métropolitains sont généralement plus longues que celles effectuées vers des lieux d'emploi situés en dehors de ces pôles. Plusieurs raisons peuvent expliquer ce phénomène dont notamment la qualité des emplois que l'on peut retrouver dans le centre-ville et les principaux pôles suburbains (activités du tertiaire supérieur ou encore activités manufacturières de haute technologie), tandis que les emplois de commerce ou de service aux personnes auraient plutôt tendance à être sur-représentés en dehors des pôles. À Montréal, où l'on constate non seulement entre 1981 et 1996, mais aussi entre 1996 et 2001, une tendance au renforcement du poids relatif des principaux pôles d'emploi (Coffey et shearmur, 2001; Shearmur et Coffey, 2002a, 2002b; Dubé, 2005; Terral et al., 2006; Shearmur et al, 2007), au sein desquels on trouve plutôt des emplois du tertiaire moteur (centre-ville) ou encore du manufacturier de haute technologie (Ville-St-Laurent-Dorval), nous faisons donc l'hypothèse que la nouvelle dynamique spatiale de l'emploi favorise plutôt une augmentation des distances de navette parcourues par les travailleurs. Et donc, corrélativement, que l'hypothèse de colocalisation s'avère inexacte, dans le cas de Montréal également.

S'agissant de la question des modes de transport, les études empiriques montrent clairement que la suburbanisation de l'emploi se fait au détriment de l'utilisation des modes de transport alternatifs, l'automobile devenant le moyen de transport privilégié par les travailleurs de ces nouveaux pôles d'emploi en plein essor. De nombreuses études, en effet, ont montré que le développement des pôles de banlieue est massivement associé à l'utilisation de l'automobile, et cela, tant en Amérique du Nord (Cervero et Wu, 1993, 1997; Wachs, 1993) qu'en Europe (Clark et Kuijpers-Linde, 1994 ; Naess et Sandberg, 1995; Schwanen et al., 2001). Cervero et Wu (1993) montrent, par exemple comment, dans la Baie de San Francisco, la part des déplacements en automobile comme conducteur est inversement proportionnelle à la position des pôles dans la hiérarchie métropolitaine, les pôles les moins denses et les plus éloignés étant associés à une beaucoup plus importante utilisation de l'automobile. Les nouvelles configurations spatiales des migrations pendulaires engendrées par le desserrement intra-metropolitain de l'emploi, beaucoup plus complexes et diffuses que dans la traditionnelle ville 
monocentrique, rendent en effet la tâche plus difficile aux fournisseurs et aux aménageurs de transports alternatifs s'efforçant de concurrencer l'ubiquité de l'automobile. Par ailleurs, il ressort clairement des très nombreuses études empiriques visant à mesurer l'impact du nouvel urbanisme et des TOD, recensées par Ewing et Cervero (2001), que ces derniers n'auraient qu'une influence très limitée sur les comportements de mobilité des individus, leurs caractéristiques socio-économiques voire même leurs préférences (Handy et Clifton, 2001, Krizek 2003) constituant des déterminants bien plus importants. Reste néanmoins que toutes ces études se sont peu appliquées à mieux caractériser et identifier les différents types de zones d'emploi des travailleurs dans leur analyse des migrations pendulaires. Au regard de la littérature évoquée précédemment, nous faisons donc l'hypothèse que le desserrement de l'emploi au sein de la RMR de Montréal se fait également au détriment des modes alternatifs de transport et à mesure que l'on descend dans la hiérarchie des zones d'emploi.

\section{Données et méthodologie}

La présente recherche exploite le potentiel d'intégration des systèmes d'information géographique (SIG) afin d'arrimer deux types de bases de données que sont (1) les données d'emploi, recensées au lieu de travail de la population active occupée, issues des recensements de 1996 et 2001 de Statistique Canada et compilées à l'échelle des secteurs de dénombrement ainsi que (2) les données de déplacements pour le travail issues des enquêtes origine-destination de 1998 et 2003 de l'Agence Métropolitaine de Transport (AMT) de Montréal et géoréférencées individuellement à l'adresse des personnes. Deux éléments méritent néanmoins d'être relevés concernant les données des enquêtes origine-destination utilisées dans cette recherche. Premièrement, les distances considérées dans cette recherche sont mesurées à vol d'oiseau mais elles constituent néanmoins, selon nous, une très bonne approximation des distances réellement parcourues par les travailleurs. Apparicio et al. (2003) ont en effet montré que, à l'échelle métropolitaine, les distances euclidiennes sont fortement corrélées avec les distances réticulaires, de telle sorte que les résultats présentés ici ne devraient pas être sensiblement influencés par ces approximations. Deuxièmement, pour plus de fiabilité dans nos analyses et afin de pallier les difficultés techniques résultant d'une classification différente des déplacements intermodaux entre les enquêtes OD de 1998 et 2003, nous avons décidé de nous en tenir uniquement à une distinction entre les déplacements effectués en automobile comme conducteur et les autres modes, que nous appelons «modes alternatifs », soit les déplacements en automobile comme passager (covoiturage), en transport en commun (Métro, train, autobus), en taxi, ou encore les transports actifs (vélo et à pied).

La pertinence de notre analyse réside dans l'utilisation d'une catégorisation de zones d'emploi extrêmement fine et détaillée résultant d'une étude précédente de l'évolution de l'emploi au sein de la RMR de Montréal entre 1996 et 2001(Shearmur et al. 2007). S'appuyant à la fois sur le concept de ville monocentrique tel qu'observé par Burgess (1925) et conceptualisé par Alonso (1964) ainsi que sur le concept de ville polycentrique formalisé pour la première fois par Harris \& Ullman (1945), la RMR de Montréal y est conceptualisée comme structurée autour d'un centre-ville d'affaires (central 
business district) et plusieurs pôles d'emploi suburbains, parmi lesquels Ville-SaintLaurent-Dorval, Longueuil, Laval, Mirabel, Anjou, Marché central. Parallèlement à cette conceptualisation de base, nous recourons également à la dichotomie noyaufrange (core-frame structure) proposée par Horwood et Boyce (1959) suggérant qu'une concentration d'emplois consiste en un noyau densément développé autour duquel se développe une zone d'emploi plus diffuse, qualifiée de frange ou de pourtour. Chacun des principaux pôles d'emploi (centre-ville et suburbains) est ainsi divisé entre son noyau et sa frange. Le découpage retenu identifie également des zones d'emploi dites « secondaires » ou « isolées » qui ne rencontrent pas les critères de définition de nos principaux pôles d'emploi mais qui, sans être contiguës à ces derniers, rencontrent néanmoins les critères des zones constituant leur frange. Le tableau 1 présente la hiérarchie des zones d'emploi retenues ainsi que les critères ayant présidé à leur identification et la carte 1 (page suivante) permet de les localiser au sein de la RMR de Montréal.

Tableau 1 : Liste des zones d'emploi de la RMR de Montréal et des critères ayant présidé à leur identification

\begin{tabular}{|l|l|}
\hline Zones d'emploi & Critères d'identification \\
\hline Hyper Centre-Ville & SD contiguës ayant E $>5000$ et Ratio EPA $>1$ localisés au centre-ville \\
\hline Franges du Centre-Ville & SD contiguës à l'hyper Centre-Ville ayant E $>500$ et Ratio EPA $>1$ \\
\hline Hyper-pôles suburbains & SD contiguës hors centre-ville ayant E $>5000$ et Ratio EPA $>1$ \\
\hline Franges des hyper-pôles & SD contiguës aux hyper-pôles ayant E $>500$ et Ratio EPA $>1$ \\
\hline Pôles secondaires & au moins deux SD contiguës ayant E $>500$ et Ratio EPA $>1$ \\
\hline Pôles isolés & un seul SD ayant E $>500$ et Ratio EPA $>1$ \\
\hline
\end{tabular}

$1 \mathrm{SD}=$ Secteur de dénombrement

$2 \mathrm{E}=$ nombre d'emplois

3 Ratio EPA = ratio emploi / population active résidente

L'étude de Shearmur et al. (2007), à la suite de laquelle se situe cette nouvelle recherche, a souligné la sensibilité des analyses au découpage des zones d'emploi retenues, compte tenu de la très fine échelle spatiale utilisée. En effet, selon que les pôles d'emploi étaient identifiés en appliquant les critères évoqués précédemment en début (1996) ou en fin de période (2001), il était possible de conclure alternativement à un processus de dispersion (scatteration) ou plutôt de renforcement de la polynucléarisation de l'emploi à Montréal, ce qui traduit finalement le fait que ces phénomènes apparemment antagonistes puissent coexister au sein de l'espace métropolitain, comme nous l'évoquions précédemment. Pour plus de fiabilité, nous avons donc décidé de réaliser nos analyses des (1) distances de navette parcourues et (2) des modes de transport utilisés par les travailleurs sur les deux découpages à la fois et de reproduire également les données de croissance de l'emploi entre 1996 et 2001 associées à chacune des catégories de zones d'emploi. 
Carte 1 : Catégorisation des zones d'emploi au sein de la RMR de Montréal en 2001

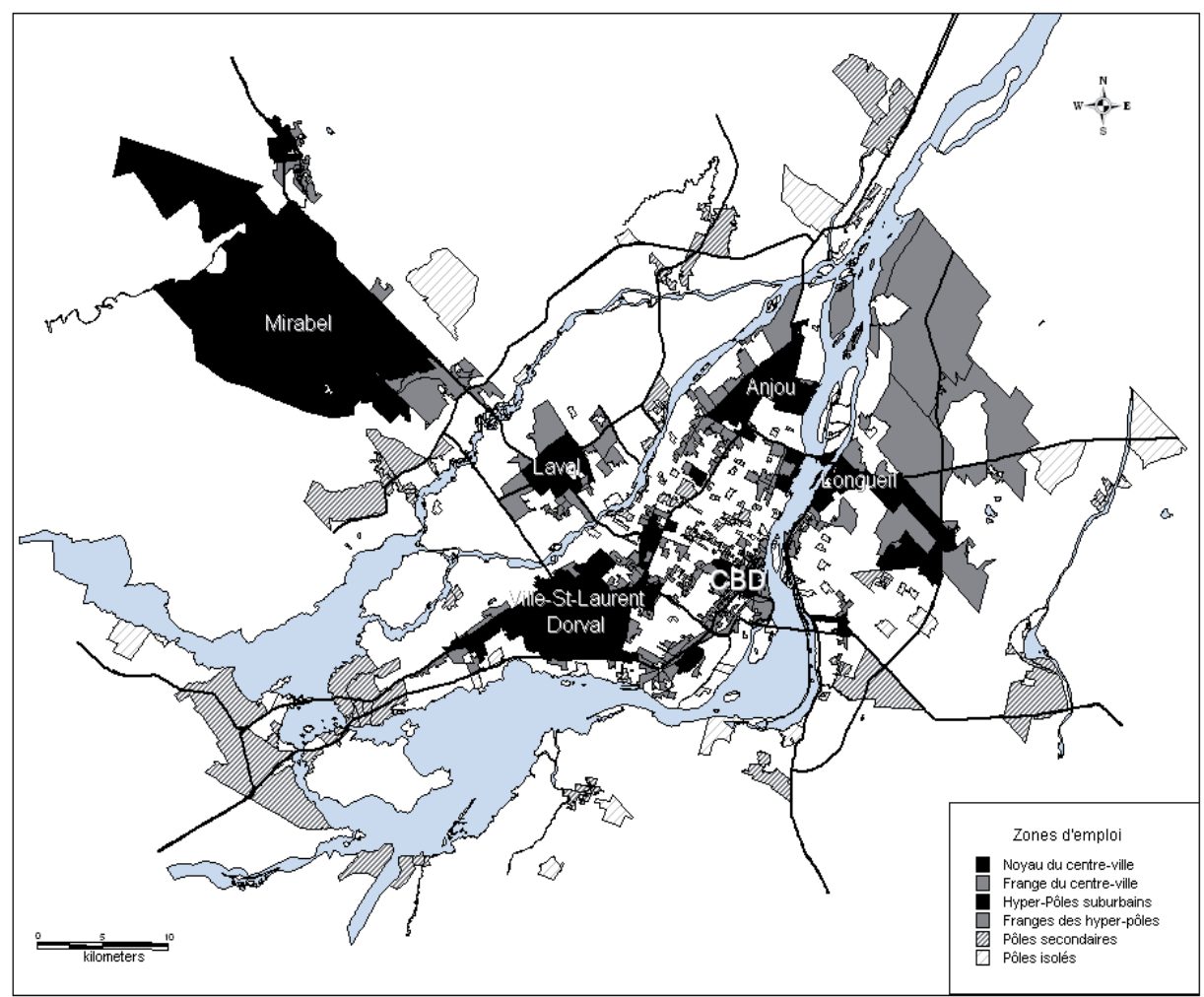

\section{Structuration spatiale de l'emploi montréalais et distances de navette parcourues}

Le tableau 2 permet de comparer les distances moyennes de navette associées à chacun des types de zones d'emploi ainsi que leur évolution entre 1998 et 2003. Cette information peut également être mise en relation avec l'importance et la croissance de l'emploi au sein de ces zones entre 1996 et 2001 ainsi qu'avec l'évolution de leur vocation, mesurée à travers un simple ratio emploi / population active résidente (EPA).

Conformément à ce que l'on retrouve dans la littérature, nous pouvons constater d'emblée que les distances de navette associées à l'ensemble des zones d'emploi sont plus longues de $2 \mathrm{~km}$ (autour de 11,5 km vs. 9,5 km) que celles associées aux emplois situés dans les zones résidentielles. Ce résultat se comprend aisément dans la mesure où ces zones d'emploi abritent généralement des emplois plus spécialisés et qualifiés, pour lesquels les travailleurs sont généralement prêts à parcourir de plus importantes distances de navette, tandis que les $30 \%$ des emplois de la RMR situés en dehors des zones d'emploi relèvent plutôt du commerce de détail et des services à la population. D'autre part, ces derniers sont beaucoup plus fortement dispersés au 


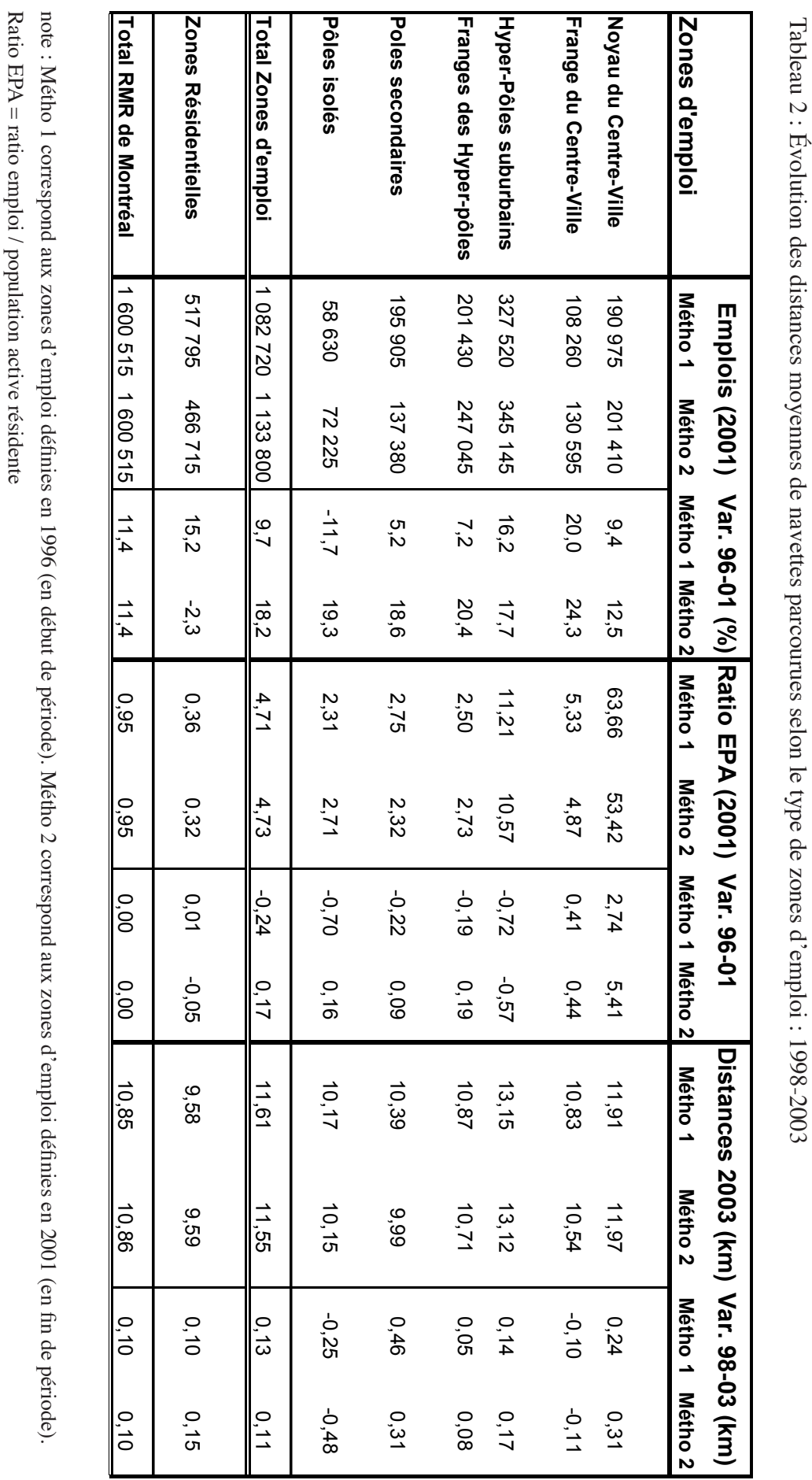


sein du tissu résidentiel comme en témoigne le très faible ratio emploi / population active résidente (ratio EPA de 0,3 contre 4,7 pour les zones d'emploi).

Mais le plus intéressant à remarquer réside dans les différences entre les types de concentrations d'emplois, d'abord en considération de leur hiérarchie mais surtout entre les noyaux des principaux pôles d'emploi et leur frange. Nous pouvons constater en effet que les noyaux des principaux pôles d'emploi gênèrent des déplacements sensiblement plus longs que le reste des zones d'emploi : près de $12 \mathrm{~km}$ dans le cas du noyau du centreville et plus de $13 \mathrm{~km}$ pour les hyper-pôles suburbains, tandis que les distances parcourues se situent autour de $10 \mathrm{~km}$ dans le cas des pôles secondaires et isolés. Mais le plus frappant réside dans les distances relativement faibles associées aux franges des principaux pôles d'emploi (du moins sensiblement moins élevées que dans leur noyau), par ailleurs quasiment identiques (variant de 10,5 km à 10,8 km selon le découpage retenu) qu'il s'agisse des franges du centre-ville ou de celles des hyper-pôles. Ce phénomène peut s'expliquer notamment par le fait que l'on trouve une beaucoup plus forte proportion de lieux de résidence dans ces franges qu'au cœur même des pôles d'emploi, comme l'attestent d'ailleurs les ratios EPA qui leur sont associés, nettement moins élevés que dans les noyaux des pôles : autour de 5 pour les franges du centre-Ville (vs. près de 60 en moyenne pour l'hyper-centre-ville) et autour de 2,5 pour les franges des hyper-pôles (vs. près de 11 en moyenne pour les hyper-pôles), soit des ratios comparables à ceux des pôles secondaires et isolés (autour de 2,5) qui sont également associés à des distances de navette sensiblement plus courtes. Ces résultats laissent donc penser que la colocalisation des lieux d'emploi et des lieux de résidence, une plus grande mixité fonctionnelle des zones donc, favorise des comportements de mobilité plus durables.

L'évolution des distances de navettes entre 1998 et 2003 vient par ailleurs confirmer cette tendance fort intéressante s'agissant des franges des principaux pôles d'emploi montréalais. En effet, tandis que les distances de navette associées aux noyaux des principaux pôles d'emploi ont tendance à augmenter (de plus de $200 \mathrm{~m}$ dans le cas du centre-ville et autour de $150 \mathrm{~m}$ dans le cas des hyper-pôles), celles associées à leurs franges respectives auraient plutôt tendance à rester stables (cas des franges des hyperpôles) voire même à diminuer (de plus de $100 \mathrm{~m}$ s'agissant des franges du centre-ville). Ce phénomène est d'autant plus remarquable qu'à l'exception des pôles isolés qui connaissent une forte diminution (de -250 à $-480 \mathrm{~m}$ ), les autres catégories de zones, y compris la zone résidentielle $(+100 \mathrm{~m})$, connaissent plutôt une augmentation des distances moyennes de navettes qui leur sont associées, augmentation parfois même très forte comme dans le cas des pôles secondaires (de 460 à $310 \mathrm{~m}$ selon le découpage retenu).

Enfin, il convient de souligner également que, à peu de choses près, le découpage de début ou de fin de période semble sans incidence sur les résultats, ce qui tend à démontrer finalement, non sans pertinence, que les distances parcourues par les travailleurs dépendent moins de la dynamique des zones d'emploi dans lesquelles ils travaillent que de la situation géographique et des attributs intrinsèques de ces zones. En effet, on voit bien (Tableau 2) que, selon le découpage retenu, la croissance de l'emploi est tantôt affectée plutôt dans les zones d'emploi tantôt plutôt dans la zone non-résidentielle, un grand nombre de secteurs de dénombrement passant entre les deux dates d'une catégorie a l'autre Or, cette différence n'affecte pas les résultats concernant les distances parcourues et leur évolution entre 1998 et 2003. 


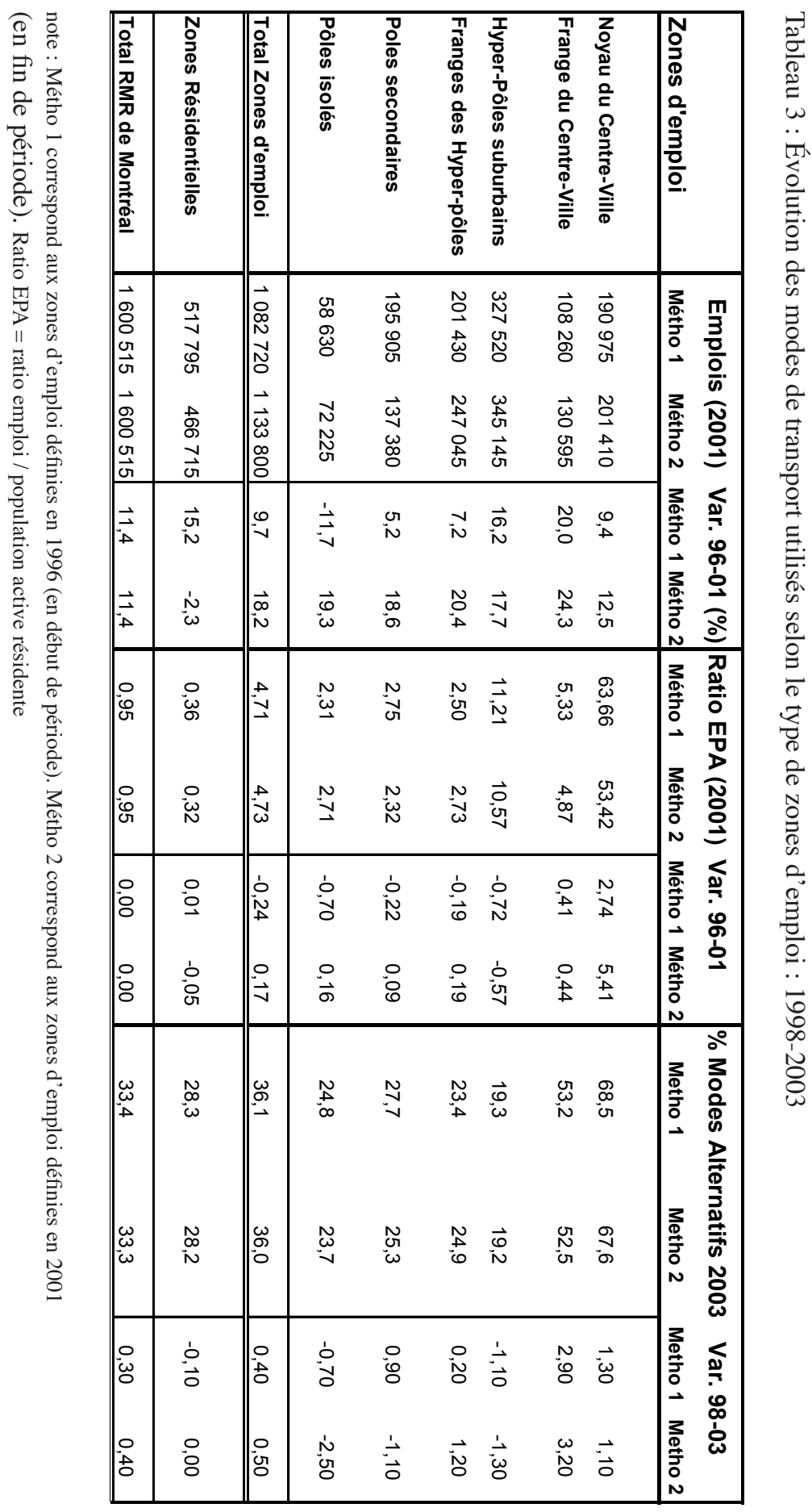


Ces résultats concernant les distances moyennes de navette associées à chacun des types de zones d'emploi ainsi que leur évolution entre 1998 et 2003 nous amènent quasiment à invalider notre hypothèse de départ (à savoir que la nouvelle dynamique spatiale de l'emploi au sein de la RMR conduit à une augmentation croissante des distances parcourues), du moins à la nuancer fortement. Certes les distances moyennes de navette associées aux principaux pôles d'emploi sont sensiblement plus longues que celles associés aux autres types de zones et continuent de s'accroître entre 1998 et 2003, de telle sorte que le renforcement de la polynucléarisation au sein de l'espace métropolitain favorise plutôt des déplacements moins « durables ». Néanmoins, le phénomène concomitant auquel on assiste, d'étalement de l'emploi dans les franges de ces pôles, conduit à nuancer fortement cette interprétation, ces dernières générant des navettes sensiblement plus courtes et par ailleurs stables ou décroissantes. Couplée à la forte diminution des distances associées aux pôles isolés, celles des franges des principaux pôles d'emploi viennent ainsi contrebalancer sensiblement l'augmentation de celles associées au noyau du centre-ville, des hyper-pôles suburbains et des pôles secondaires, de telle sorte que l'on constate une augmentation des distances parcourues de seulement 130 à $110 \mathrm{~m}$ si l'on considère globalement l'ensemble des zones d'emploi; augmentation par ailleurs comparable à celle que l'on observe au sein de la zone résidentielle (de 100 à $150 \mathrm{~m}$ ).

\section{Structuration spatiale de l'emploi montréalais et modes de transport utilisés}

Le tableau 3 présente la part des modes de déplacement alternatifs à l'automobile (covoiturage, transport en commun, taxi, vélo, à pied) associée à chacune des catégories de zones d'emploi ainsi que son évolution entre 1998 et 2003.

Sans grande surprise, on remarque d'emblée que la part des modes alternatifs est beaucoup plus importante pour le centre-ville, qu'il s'agisse de son noyau (autour de $68 \%$ selon le découpage retenu) ou de sa frange (autour de $63 \%$ ), que pour les autres types de zones d'emploi, dont la part d'utilisation des modes alternatifs varie de $19 \%$ à $30 \%$. Cette bonne performance du centre-ville s'explique bien évidemment par le fait que d'importantes infrastructures de transport collectif (comme le métro ou le train) convergent vers lui mais également par le fait que les travailleurs qui s'y rendent cherchent à éviter l'importante congestion existant aux heures de pointe. Ces résultats militent manifestement en faveur du modèle de la ville compacte comme moyen d'encourager l'utilisation du transport en commun.

Parmi les autres zones d'emploi, il convient de souligner que ce sont surtout les hyper-pôles qui génèrent le plus de déplacements en automobile, la part des modes alternatifs n'atteignant même pas les $20 \%$. Toutefois, ce pourcentage atteint les $24 \%$ pour tous les déplacements effectués dans leur frange. Une explication possible de cette différence marquée résiderait dans le fait que ces franges particulièrement dynamiques sont généralement structurées le long des principaux axes de communication ou existent des solutions de transport alternatives à l'automobile, comme le transport en commun ou parfois même des pistes cyclables, quand certains hyper-pôles ne sont même pas inclus dans l'un de ces réseaux (Ville-Saint-Laurent-Dorval).

Avec une proportion située autour de $25 \%$, les pôles secondaires et isolés semblent toutefois légèrement plus à l'avantage des modes alternatifs que ne le sont les principaux 
pôles suburbains. Enfin, la zone résidentielle est associée à des proportions de modes alternatifs légèrement plus élevés encore $(28 \%)$ mais néanmoins toujours beaucoup plus faibles que dans le centre-ville. Force est donc de constater que, même si le centre-ville s'avère largement plus à l'avantage des modes de transport alternatifs, leur utilisation ne décroît pas à mesure que l'on descend dans la hiérarchie des zones d'emploi.

Il est intéressant de souligner également que la part des déplacements alternatifs varie peu entre 1998 et 2003 à l'échelle de la région métropolitaine mais qu'en revanche on observe de plus fortes variations dès lors que l'on commence à distinguer entre les différentes catégories des zones d'emploi. En effet, nos résultats montrent que la part des transports alternatifs progresse encore dans le centre-ville, gagnant plus de un point de pourcentage dans le noyau central mais surtout dans ses franges où la progression est de l'ordre de 3 points de pourcentage, ce qui est assez considérable sur une période de 5 ans. En revanche, la tendance est inverse s'agissant des pôles de banlieue, qu'il s'agisse des hyper-pôles $(-1,3$ points de pourcentage) ou des pôles secondaires $(-1,1)$ et isolés $(-2,5)$. À l'instar de ce que l'on observe dans le centre-ville, la part des modes alternatifs augmente néanmoins dans les franges des hyper-pôles $(+1,2)$.

Finalement, nos résultats invalident notre hypothèse de départ voulant que le desserrement de l'emploi métropolitain montréalais se fasse au détriment des modes alternatifs de transport et à mesure que l'on descend dans la hiérarchie des zones d'emploi. Certes le renforcement de la polynucléarisation à Montréal se fait au profit de l'automobile, mode de transport privilégié par les travailleurs des principaux pôles suburbains, qui par ailleurs parcourent aussi des distances plus importantes. Néanmoins, le centre-ville et surtout ses franges ainsi que celles des hyper-pôles, dont nous savons qu'elles captent une part importante de la croissance de l'emploi entre 1996 et 2001, favorisent l'utilisation des modes de transports alternatifs à l'automobile. C'est d'ailleurs la raison pour laquelle on observe une légère tendance à la progression des modes alternatifs si l'on considère l'ensemble des zones d'emploi de la RMR de Montréal, tandis que la situation reste stable pour ce qui est des zones résidentielles.

\section{Desserrement de l'emploi et migrations pendulaires plus « durables»? : des tendances simultanées conduisant à un bilan nuancé}

La variabilité de nos résultats (tant au niveau des distances parcourues que des modes de transport utilisés) selon le type de zone d'emploi, alors qu'à un niveau plus agrégé les différences ont tendance à être beaucoup moins marquées, souligne toute la pertinence de recourir à une échelle d'analyse spatiale et une classification plus fine et détaillée des zones d'emploi que celles adoptées généralement dans la littérature. Une approche à plus petite échelle permet ainsi de mettre en lumière l'hétérogénéité qui peut exister au sein des principaux pôles d'emploi, hétérogénéité masquant des comportements de mobilité forts différents. En effet, tandis que les noyaux des principaux pôles d'emploi sont associés à la fois à des distances plus longues et à une utilisation ultra-dominante de l'automobile comme conducteur (à l'exception du centre-ville toutefois), les franges de ces pôles, qui connaissent par ailleurs un fort dynamisme sur le plan de l'emploi, sont associées quant à elles à de plus courtes distances et à une utilisation plus importante des modes de transport alternatifs à l'automobile. De plus, nos 
résultats indiquent que ces différences entre les noyaux et les franges se renforcent assez sensiblement entre 1998 et 2003. Ainsi, si à une échelle spatiale d'analyse relativement agrégée, la plupart des études concluent que la polynucléarisation de l'emploi va de pair avec l'adoption de comportements de mobilité moins durables, l'utilisation d'un découpage plus raffiné des zones d'emploi, distinguant notamment entre les noyaux et les franges des principaux pôles d'emploi révèle que cette tendance est largement contrebalancée par les nouvelles formes d'étalement de l'emploi autour de ces pôles, le plus souvent le long des principaux axes de transports et les reliant entre eux. Ces résultats sont d'autant plus intéressants que, comme nous l'avons évoqué précédemment, les franges des principaux pôles d'emploi présentent une plus forte mixité fonctionnelle, comprenant un ratio emploi / population active résidente nettement plus équilibré que dans le cas des noyaux de ces pôles. Ces résultats laissent donc penser que la colocalisation des lieux d'emploi et des lieux de résidence, favorise effectivement des comportements de mobilité plus durables, notamment du fait que ces franges se structurent le long des principaux axes de communication, où existent des réseaux de transport en commun et parfois même des pistes cyclables.

Finalement, la spécificité et la «performance » des franges des pôles (centre-ville et hyper-pôles) invite à s'interroger sur la pertinence d'une structuration axiale, plutôt que multipolaire, des activités et des déplacements à l'échelle métropolitaine. Shearmur et al (2007) ont déjà souligné que l'analyse de la répartition intra-métropolitaine de l'emploi à très fine échelle spatiale d'analyse :

" suggests an alternative way of conceptualising the distribution of employment in intrametropolitan space, one that draws upon the concepts of employment "poles" or "centres", but also upon economically important physical links between the centres. It is no longer just the "edge cities" and suburban centres that are important, but the "spaces in-between" ".

D'autres études ont également permis de souligner la pertinence d'une structuration axiale des activités, particulièrement au sein de métropoles de plus petite taille (Villeneuve et al, 2006; Barbonne et al. 2003). Plus largement, c'est également la question corrélative des déplacements qui gagnerait également à être repensée dans cette perspective. Les axes permettent la diffusion spatiale des économies d'agglomération, parfois même au-delà des frontières métropolitaines (Barbonne, 2006). Peut-être même d'ailleurs est-ce le long de ces axes que s'organisent ces fameuses « edgeless cities » conceptualisées par Lang (2003)? Plus largement encore, on peut se demander si ces axes ne permettent pas également une gestion plus rationnelle et plus efficace des ressources. Les axes présentent en général une plus forte diversité une plus grande mixité des fonctions, de telle sorte qu'un corridor urbain assez dense et multifonctionnel constitue probablement la meilleure condition pour favoriser le transport en commun et sa rentabilité : les véhicules connaissent en effet de meilleurs taux de remplissage dans les deux sens, ce qui n'est pas le cas d'un pôle qui se remplit le matin et se vide le soir. Un ensemble d'axes formant un réseau à l'échelle du territoire de la ville constitue par ailleurs un excellent moyen pour penser l'intégration du territoire : ceux-ci unissent les parties du territoire, à l'opposé des pôles qui veulent, chacun, attirer le développement. Enfin, La mixité des fonctions complémentaires le long d'un axe fait en sorte que les diverses parties du territoire traversées par l'axe profitent des retombées indirectes d'investissement fait le long de l'axe, même si elles sont à plusieurs kilomètres de l'endroit où se fait l'investissement car l'axe crée des liens fonctionnels. 
Cette étude atteste donc de la pertinence de prendre en compte, plus finement qu'on ne le fait généralement, la localisation des emplois, le type de zones dans lesquelles la population active occupée se rend travailler. Néanmoins, pour consolider ces résultats, il conviendrait de pouvoir tester plus spécifiquement l'influence de cette fine localisation de l'emploi dans la structure explicative des migrations pendulaires, en maintenant constant un plus grand nombre de paramètres. Des paramètres rattachés au lieu de résidence et au voisinage, comme par exemple les revenus, le niveau de qualification, le sexe, l'âge, les responsabilités familiales, la présence d'enfants au sein des familles de ces navetteurs, etc. Des paramètres rattachés au lieu de travail aussi, comme par exemple la spécialisation sectorielle et la mixité fonctionnelle des concentrations d'emploi, leur distance du centreville, etc. Plus largement, se pose la question de savoir dans quelle mesure la problématique des migrations pendulaires n'est pas seulement un arrangement quantitatif entre des « masses » mesurées aux lieux de travail et aux lieux de résidence, tel que conçu dans le modèle gravitaire ou à travers le concept de «job-houses imbalances », mais bien plus un appariement qualitatif entre certains types d'emplois et certains profils de travailleurs, tel qu'investigué à travers la question du « spatial mismatch » (Kain, 1968; Preston et McLafferty, 1999; Houston, 2005). Par ailleurs, il conviendrait également de s'interroger plus largement sur les chaînes de déplacements, autant s'agissant des déplacements intermodaux que des déplacements pour autres motifs (commerces notamment) qui très souvent s'organisent autour des migrations pendulaires (Kwan, 1999; Dielman et al. 2002) et voir, plus spécifiquement, dans quelle mesure les déplacements pour le travail dans les franges des principaux pôles d'emploi favorisent également l'achat concomitant de biens et services.

\section{Remerciements}

Les auteurs remercient l'Agence Métropolitaine de Transport (AMT) de Montréal pour la fourniture des données des enquêtes Origine-Destination de 1998 et 2003 et plus particulièrement M. Daniel Bergeron pour son soutien technique. Cette recherche a également été rendue possible par le biais de financements provenant du Conseil de recherches en sciences humaines du Canada (CRSH). 


\section{Références}

Alonso, W. (1964) Location and Land Use, Cambridge (MA) : Harvard University Press.

Apparicio, P., Shearmur, R., Brochu, M. et Dussault, G. (2003) « The Measure of Distance in a Social Science Policy Context : Advantages and Costs of Using Network Distances in 8 Canadian Metropolitan Areas », Journal of Geographic Information and Decision Analysis, 7, 105-131.

Aguilera, A., et Mignot, D. (2002) «Structure des localisations intra-urbaines et mobilité domicile-travail », Recherche Transport, Sécurité, 77, 311-325.

Aguilera, A. (2005) « Growth in Commuting Distances in French Polycentric Metropolitan Areas : Paris, Lyon and Marseille », Urban Studies, 42, 1537-1547.

Barbonne, R., Bourel C., Robin J. et Villeneuve P. (2003) Le territoire d'intervention de la CMQ : ses limites et son intégration. Étude réalisée pour la Communauté métropolitaine de Québec, CRAD, Université Laval.

Barbonne, R. (2006) Pôles d'emploi, bassins de main-d'œuvre et disparités locales de revenu au sein du champ métropolitain de Québec : 1980-2000, Sainte-Foy, Université Laval, Thèse de doctorat.

Barbonne, R., Villeneuve, P. et Thériault, M., (2007). « La dynamique spatiale des bassins de main-d'œuvre au sein du champ métropolitain de Québec : 1981-2001 », Le Géographe Canadien, 51(3), 303-322.

Bogard, W.T. et Ferry W.C. (1999) «Employment Centres in Greater Cleveland : Evidence of Evolution in a Formely Monocentric City », Urban Studies, 36, 2099-2110.

Buchanan N., Barnett, R., Kingham, S. et Johnston, D. (2006) « The Effect of Urban Growth on Commuting Patterns in Christchurch, New Zealand », Journal of Transport Geography, 14, 342-354.

Burgess, E. (1925) «The Growth of the City», in The City, ed. R.Park, E.Burgess and R.McKenzie, Chicago : Chicago University Press, 37-44.

Calthorpe, P. (1993) The Next American Metropolis : Ecology, Community and the American Dream, Princeton Architectural Press, New-York.

Cervero R. et Wu K-L. (1997) « Polycentrism, Commuting, and Residential Location in the San Francisco Bay Area », Environment and Planning A, 29, 865-886.

Cervero R. et Wu K-L. (1998) «Subcentering and Commuting : Evidence from the San Fransisco Bay Area», Urban Studies, 35, 1059-1076.

Cervero R. et Kockelman, K. (1997) «Travel Demand and the 3Ds : Density, Diversity and Design », Environment and Planning D, 2, 199-219.

Coffey, W. J. et Shearmur, R. (2001) «Intrametropolitan Employment Distribution in Montreal, 1981-1996», Urban Geography, 22, 106-129.

Coffey, W. J. et Shearmur, R. (2002) «Agglomeration and Dispersion of High-order Service Employment in the Montreal Metropolitan Region, 1981-1996», Urban Studies, 39, 359-378.

Clark, W.A. et Kuijpers-Linde, M. (1994) « Commuting in Restructuring Urban Regions », Urban Studies, 31, 465-483.

Dear, M. J. et Flusty, S., eds. (2001) The Spaces of Postmodernity, Oxford, Blackwell.

Dielman, F., Djist, M. et Burghouwt, G. (2002) « Urban Form and Travel Behavior : Micro-level Household Attributes and Residential Context », Urban Studies, 39, 507-527.

Ewing, R. et Cervero, R. (2001) «Travel and the Built Environment. A Synthesis», Transportation Research Record, 1780, 87-113.

Dubin, R. (1991) «Commuting Patterns and Firm Decentralization », Land Economics, 67, 15-29.

Fuji, T. et Hartshorn, T.A. (1995) « The Changing Metropolitan Structure of Atlanta, Georgia : Locations of Functions and Regional Structure in a Multinucleated Urban Area », Urban Geography, 16, 680-707.

Garreau J. (1991) Edge City : Life on the New Frontier, New York : Anchors Books.

Gordon, P., Kumar, A. et Richardson, H.W. (1989) « Congestion Changing Metropolitan Structure, and City Size in the US », International Regional Science Review, 12, 45-56. 
Gordon, P., Richardson, H.W. et Jun, M.-J. (1991) «The Commuting Paradox : Evidence from the Top Twenty », Journal of the American Planning Association, 57, 416-420.

Gordon, P. et Richardson, H.W. (1996) « Beyond Polycentricity, The Dispersed Metropolis, Los Angeles, 1970-1990 », Journal of the American Planning Association, 62, 289-295

Giuliano G. et Small, K.A. (1991) «Subcenters in the Los Angeles Region », Regional Science and Urban Economy, 21, 163-182.

Giuliano G. et Small, K.A. (1993) « Is the journey-to-work explained by urban structure? », Urban Studies, 30, 1485-1500.

Harris, C et E.Ullman (1945) The Nature of Cities, Annals of the American Academy of Political and Social Science, 242, 7-17, reproduced in Putnam.R,, Taylor.F, and Kettle.P., eds, (1970) A Geography of Urban Places, London : Methuen, 91-101.

Hartshorn, T.A., et Muller, P.O. (1989) « Suburban downtowns and the transformation of metropolitan atlanta's business landscape », Urban Geography, 10, 375-395.

Horwood, E. M.et Boyce, R. R. (1959) Studies of the Central Business District and Urban Freeway Development, Seattle : University of Washington Press.

Houston, D. (2005) « Employability, Skills Mismatch and Spatial Mismatch in Metropolitan Labour Markets», Urban Studies, 42, 221-243.

Kain, J.F. (1968) « Housing Segregation, Negro Unemployment, and Metropolitan Segregation », Quarterly Journal of Economics , 82, 175-197.

Kelbaugh, D., ed. (1989) The Pedestrian Pocket Handbook : a New Suburban Design Strategy, Princeton Architectural Press, New-York.

Kwan M-P. (1999) « Gender, The Home-work Link and Space-time Patterns of Non-employment Activities», Economic Geography, 75, 370-394.

Lang, R.E. (2003) Edgeless Cities : Exploring the Elusive Metropolis, Washington : The Brookings Institution. Levinson, D. M. (1998) «Accessibility and the Journey to Work », Journal of Transport Geography, 6, 11-21.

McDonald, J. et Prather, P. (1994) «Suburban Employment Centres : the Case of Chicago », Urban Studies, 31, 201-218.

Naess, P. et Sandberg, S.L. (1995) «Workplace Location, Modal Split and Energy Use for Commuting Trips», Urban Studies, 33, 557-580.

Newman P. et Kenworthy, J. (1996). « The Land Use-Transport Connection. An Overview », Land Use Policy, $13,1-22$.

Newman P. et Kenworthy, J. (1999) Sustainability and Cities : Overcoming Automobile Dependence, Washington : Island Press.

Pfister, N., Freestone, R. et Murphy, P. (2000) « Polycentricity or Dispersion ?: Changes in Center Employment in Metropolitan Sydney, 1981 to $1996 »$, Urban Geography, 21, 428-442.

Polèse, M. et Chapain, C. (2000) Are Central CBDs Really Declining? An Analysis for US and Canadian Urban Areas, Montréal : INRS-Urbanisation, Culture et Société, collection Inédit, 2000-2.

Preston, V. et McLafferty, S. (1999) « Spatial mismatch research in the 1990s : progress and potential », Papers in Regional Science, 78, 387-402.

Rosetti, M. et Eversole, B. (1993) Journey to Work Trends in the United States and its Major Metropolitan Areas, 1960-1990, Washington D.C. : Department of Transportation.

Schwanen T., Dieleman,F.M. et Dijst, M. (2004) « The Impact of Metropolitan Structure on Commute Behavior in the Netherlands : a Multilevel Approach », Growth and Change, 35, 304-333.

Shearmur, R. et Coffey, W. J. (2002a) « A Tale of Four Cities : Intrametropolitan Employment Distribution in Montreal, Toronto, Vancouver and Ottawa, 1981-1996 », Environment and Planning A, 34, 575-598.

Shearmur, R. et Coffey W. J. (2002b) « Urban Employment Sub-centres and Sectoral Clustering in Montreal : a Complementary Approach to the Study of Urban Form », Urban Geography, 23, 103-130. 
Shearmur, R., 2006. « Travel-from-home; an Economic Geography of Commute Distances in Montreal », Urban Geography, 27, 330-359.

Shearmur, R., Coffey, W., Dubé, C. et Barbonne, R. (2007) « Intrametropolitan Employment Structure : Polycentricity and Scaterration in Toronto, Montreal and Vancouver, 1996-2001», Urban Studies, 44, 1713-1738.

Stanback, T.M. (1991) The New Suburbanisation : Challenges to the Central City, Boulder : Westview Press.

Sultana, S. (2000) « Some Effects of Employment Centers on Commuting Times in the Atlanta Metropolitan Area, $1990 »$, Southeastern Geographer, 41, 225-233.

Terral, L. et Shearmur, R. (2006) « Structures et logiques du redéploiement de l'emploi métropolitain », Territoires en Mouvement, 2.2006, 48-56.

Villeneuve, P., Kestens, Y., Barbonne, R., Robin, J. et Bourel, C. (2006) « Exploring Alternatives to Sprawl in the Quebec Metropolitan Area », in Cabana R. and Wagner F. (ed.) The International Faces of Urban Sprawl. Lessons Learned from North America, Waterloo, University of Waterloo, Department of Geography Publication Series, 62, 19-50.

Wachs, M., Taylor, B.D., Levine, N. et Ong, P. (1993) «The Changing Commute : A Case Study of the JobsHousing Relationship over Time », Urban Studies, 30, 1711-1729. 